doi: http://dx.doi.org/10.5892/ruvrv.2013.111.5867

\title{
Avaliação do conhecimento sobre os contraceptivos orais entre as universitárias
}

\author{
Thais Baptistella FELIPE ${ }^{1}$ \\ Patrícia Teatin JULIATO ${ }^{2}$ \\ Samir Antonio Rodrigues ABJAUDE ${ }^{3}$ \\ Nicole Rodrigues da SILVA ${ }^{1}$ \\ Ricardo Radighieri RASCADO ${ }^{4 *}$
}

\begin{abstract}
${ }^{1}$ Acadêmicas da Faculdade de Ciências Farmacêuticas. Universidade Federal de Alfenas, Minas Gerais. Brasil. Email:thaisfelipe@hotmail.com; nikarodrigues963@hotmail.com.

${ }^{2}$ Mestranda em Biociências e tecnologia de produtos Bioativos. Universidade Estadual de Campinas, São Paulo. Brasil. E-mail: patriciajuliato@gmail.com

${ }^{3}$ Mestrando da Faculdade de Ciências Farmacêuticas de Araraquara. Universidade Estadual Paulista "Júlio de Mesquita Filho", São Paulo. Brasil. E-mail: samirabjaude@ hotmail.com

${ }^{4}$ Doutor Professor de Atenção Farmacêutica da Faculdade de Ciências Farmacêuticas. Universidade Federal de

Alfenas, Minas Gerais. Brasil. E-mail: ricardounifal@gmail.com
\end{abstract}

*AUTOR CORRESPONDENTE: Dr. Ricardo Radighieri Rascado

Endereço: Universidade Federal de Alfenas, UNIFAL-MG, Campus Alfenas, Rua Gabriel Monteiro da Silva, 700, 37130-000. Alfenas-MG, Brasil. E-mail: ricardounifal@gmail.com

\section{Recebido em: 13/05/2013 - Aprovado em: 11/07/2013 - Disponibilizado em: 15/08/2013}

\section{RESUMO}

As técnicas usadas para o controle de natalidade avançaram consideravelmente nos últimos anos, devido o avanço da medicina e, hoje, existem muitos tipos de anticoncepcionais à base de hormônios disponíveis no mercado brasileiro que podem eventualmente gerar muitas dúvidas em relação a sua utilização. A análise do conhecimento sobre métodos anticoncepcionais, na maioria dos estudos disponíveis é feita de maneira muito subjetiva e isso pode produzir uma interpretação não verdadeira do grau de conhecimento sobre prevenção de gravidez. Este estudo teve como objetivo analisar a diferença de conhecimento e atitude das universitárias das áreas da saúde, exatas e humanas sobre as indicações e contraindicações dos contraceptivos orais. Além disso, pretendeu analisar se houve um aumento no conhecimento durante o curso de graduação, comparando as alunas do primeiro e do último período das áreas citadas. Tratou-se de um estudo observacional, transversal, descritivo e foi utilizado um questionário semiestruturado autoaplicável. O estudo foi aprovação com o número do processo 088/2010 no Comitê de Ética em Pesquisa da UNIFAL-MG e teve duração de agosto de 2010 a julho de 2011. Foram analisados 302 questionários respondidos por acadêmicas da UNIFAL-MG. Em relação às acadêmicas entrevistadas, 49,3\% fazem uso de anticoncepcional oral. No entanto, não houve uma diferença considerável entre as diferentes áreas e nem entre os períodos. Demonstrando que apesar das universitárias apresentarem melhores condições de vivenciar a sexualidade de forma mais segura e sem riscos a sua saúde, o segmento mais escolarizado também se depara com inconsistências no uso de métodos contraceptivos orais.

Palavras-chave: Anticoncepcionais. Conhecimento. Toxicidade de Drogas. Farmacovigilância. Saúde da Mulher.

\begin{abstract}
The techniques used for birth control have advanced considerably in recent years due to advances in medicine, and today there are many types of hormone-based contraceptives available in the market that can generate many questions about its use. The analysis of knowledge of contraceptive methods, in most studies is made available in a very subjective and it cannot produce a true interpretation of the degree of knowledge about pregnancy prevention. This study aimed to analyze the difference in knowledge and attitude of the university in the areas of health, human and exact about the indications and contraindications of oral contraceptives. Furthermore, we intended to examine whether there was an increase in knowledge during the undergraduate course, comparing the students of the first and last period of the areas mentioned. This was an observational, cross-sectional, descriptive and used a self-administered semistructured questionnaire. The study was approved with the case number 088/2010 on Ethics in Research UNIFAL-MG and lasted from August 2010 to July 2011. We analyzed 302 questionnaires completed by the academic UNIFAL-MG. Regarding academic respondents, $49.3 \%$ use of oral contraceptives. However, there was a considerable difference between different areas and between both periods. Demonstrating that despite the university have better conditions of
\end{abstract}


living sexuality more safely and without risks to their health, the more educated segment is also faced with inconsistencies in the use of oral contraceptives.

Keywords:Contraceptive Agents. Knowledge. Drug Toxicity. Pharmacovigilance. Women's Health.

\section{INTRODUÇÃO}

O Planejamento Familiar que consta na Constituição Federal e na Lei n ${ }^{\circ}$ 9.263, de 12 de janeiro de 1996, permitiu que as pessoas decidissem quantos filhos gostariam de ter, quando desejariam e em qual momento da vida do casal isso deveria acontecer, além disso, esse planejamento deu o direito ao casal de possuir informações de como ter filhos ou de como prevenir uma gravidez indesejada (BRASIL, 2006 citado por SEABRA et al., 2012).

Nos últimos 10 anos verificaram-se que as mulheres estão começando sua vida sexual cada vez mais cedo, e o mesmo sucedendo com a prática contraceptiva (FRANCO et al., 2008). No entanto, se esta contracepção fosse feita de forma incorreta poderia levar a danos a saúde da mulher como abortamento da criança ou até o óbito da gestante (SOUZAet al., 2006 citado por SEABRA, et al., 2012).

Os contraceptivos orais (CO) representaram o método reversível com maior utilização em um estudo nos Estados Unidos. Ainda assim, houve cerca de setecentos e cinquenta mil gestações não planejadas, pressupondo que o uso incorreto do contraceptivo levou a inefetividade deste método (HENSHAW citado por TRUSSELL, VAUGHAN, 1999).

Segundo Martins e colaboradores (2006), após analisarem vários estudos sobre conhecimento de $\mathrm{CO}$, verificaram que os estudos eram muitos subjetivos, não acrescentando a indicação, a contraindicação, o modo de administração e o efeito colateral do CO. Isso poderia causar uma interpretação equivocada do nível de conhecimento sobre os métodos preventivos da gravidez na adolescência, levando a uma analise tendenciosa no conhecimento sobre os métodos anticoncepcionais.

Diante disso, o presente projeto pretendeu comparar a atitude e o conhecimento sobre os contraceptivos orais entre as estudantes dos diferentes cursos e períodos da Universidade Federal de AlfenasMG.

usuárias de $\mathrm{CO}$ e prevalência de atitude e conhecimento sobre os contraceptivos orais entre as universitárias das diferentes áreas e 
Local do estudo - O estudo foi desenvolvido na Universidade Federal de Alfenas (UNIFAL-MG), campus Alfenas.

População e Amostra- A UNIFALMG contava em 2010 com aproximadamente 2.716 discentes no campus de Alfenas-MG, dentre os quais a população feminina de discentes era 1.779, distribuídos entre os cursos. Foram selecionados apenas discentes do sexo feminino do primeiro e último período dos cursos de graduação da UNIFALMG e utilizou como critério de exclusão a recusa de participar do estudo. A partir disso, foi calculado o tamanho amostral segundo o tamanho populacional, considerando o total de acadêmicas da instituição. Portanto, a amostra necessária para ter $95 \%$ de confiança, e $0,05 \%$ de erro era de no mínimo 316 alunas. A amostra foi constituída de alunas do primeiro e do último período, divididas em três grupos: área da saúde (alunas de farmácia, ciências biológicas, nutrição e odontologia), área de exatas (alunas de química, matemática, física e biotecnologia) e área de humanas (alunas de história, letras, pedagogia e geografia).

Período de estudo - O período de coleta ocorreu entre os meses de agosto a dezembro de 2010, mas o estudo teve duração de um ano, finalizando em julho de 2011.

\section{Instrumentos e técnicas de pesquisa}

- Inicialmente foi realizado um estudo piloto com acadêmicas do primeiro e o último período de um curso a fim de validar o questionário. Após isso, as acadêmicas foram abordadas dentro das salas de aula, informadas sobre a pesquisa e questionadas sobre o interesse em responder o questionário semiestruturado autoaplicável, que investigava o perfil farmacoepidemiológico e conhecimento e atitude sobre contraceptivo oral. O questionário era composto de perguntas objetivas dividido em duas partes. Sendo a primeira parte com perguntas para caracterizar a amostra, como idade, número de filhos, curso de graduação, uso de contraceptivo oral, motivo do uso, suspensão do medicamento, métodos contraceptivos, hábito de fumar, patologias, quem indicou o anticoncepcional, adesão ao medicamento, métodos para lembrar e o nome do contraceptivo oral que utilizava. A segunda parte do questionário eram perguntas baseadas nas bulas dos anticoncepcionais para avaliar o conhecimento sobre estes medicamentos: $\quad$ se o anticoncepcional engorda; se poderia causar dificuldade de engravidar; se é necessário proporcionar um descanso entre as cartelas; quais seriam as classes de medicamentos que poderiam interferir na ação de alguns contraceptivos orais; se haveria efetividade do contraceptivo oral se ocorresse vômito ou diarreia; e detectar as suspeitas de possíveis eventos adversos ao CO.

Aspectos éticos - $\mathrm{O}$ estudo foi aprovação com o número do processo 088/2010 no Comitê de Ética em Pesquisa da UNIFAL-MG. As acadêmicas foram 60 
voluntária e aquelas que aceitassem em participar do estudo assinaram o termo de

\section{RESULTADOS}

Foram analisados 302 questionários, sendo 136 na área da saúde, 75 na área das ciências exatas e 91 na área das ciências humanas. $\mathrm{O}$ número de questionários aplicados foi inferior ao obtido pela amostragem devido ao baixo número de universitárias que cursavam a área de humanas e de exatas.

A idade de 17 a 20 anos foi predominante entre as universitárias correspondendo $48,3 \%$, seguida de $37,1 \%$ pelas universitárias de 21 a 25 anos e por último 14,6\% possuíam acima de 25 anos. Sendo que $90,7 \%$ delas não possuíam filhos.

Cerca de 49,3\% (n=149) das acadêmicas tomavam a pílula anticoncepcional e apenas $24,2 \%$ nunca utilizaram esse método contraceptivo. Em relação ao tempo de uso do $\mathrm{CO}, 24,2 \%$ usavam a menos de um ano; $37,6 \%$ de um a três anos; $22,1 \%$ entre três a cinco; $13,4 \%$ entre cinco a dez anos e apenas $2,7 \%$ usavam a mais de 10 anos.

Entre as universitárias que nunca tomaram pílula anticoncepcional e as que pararam de tomar representam 50,7\% $(n=153)$, estas relataram que o motivo mais frequente foi que não vivenciaram uma vida sexual $41,7 \% \quad(n=63), 15,2 \% \quad(n=23)$ utilizavam outro método e $11,9 \% \quad(n=18)$ relataram sofrerem de reações adversas ao consentimento livre e esclarecido (TCLE).

anticoncepcional. Sobre a indicação do contraceptivo oral $93,9 \%$ das acadêmicas responderam que a prescrição do método contraceptivo foi feito pelo médico.

O principal motivo que levava as universitárias a utilizarem a pílula contraceptiva oral era o de evitar a gravidez $31,8 \%$ ( $\mathrm{n}=99)$. Seguido pelo motivo de regular o ciclo menstrual $26,4 \%$, evitar tensão pré-menstrual e cólica $18,0 \%$, tratamento de ovário policístico $12,9 \%$, e por último o tratamento de acnes $11 \%$. Dentro o grupo das acadêmicas que utilizavam o $\mathrm{CO}$, pode perceber que $31,7 \%$ não utilizavam outro método e $16,8 \%$ às vezes utilizava outro método, como o método de barreira.

Ao traçar o perfil das universitárias que utilizavam o $\mathrm{CO}$ notou-se que $16,1 \%$ apresentavam varizes; apenas $6,0 \%$ das universitárias fumavam; 2,0\% apresentavam problemas cardiovasculares e $6,0 \%$ problemas renais. A maioria possuía hábitos saudáveis, como o de não fumar, e também não apresentavam doenças crônicas como hipertensão arterial, diabetes mellitus, varizes, problemas renais e doenças cardiovasculares.

Quanto a pontualidade no horário de tomar os comprimidos $81,2 \% \quad(n=121)$ das entrevistadas responderam que tomavam o CO no mesmo horário todos os dias, sendo 
que $34,9 \%(n=52)$ utilizavam o despertador para lembrar.

Ao serem questionadas sobre quantos comprimidos esqueciam de tomar por cartela,28,8\% ( $n=43)$ esqueciam no máximo um comprimido, $12,7 \%$ dois comprimidos e $2,7 \%$ esqueciam de três a cinco comprimidos.

Quando questionado as universitárias sobre a confiança no método $\mathrm{CO}$, observou que na área de saúde a média foi $42,2 \%$ das universitárias confiavam no contraceptivo oral; na área de humanas a média foi 38,0\%; e na área de exatas a média foi $31,0 \%$. Além disso, constatou uma maior diferença na variação de confiança neste método pelas acadêmicas da área de saúde, variando de 25,6\% nos primeiros períodos para 58,7\% nos últimos períodos.

A segunda parte do questionário teve como objetivo verificar se havia diferenças no nível de conhecimento sobre anticoncepcional oral entre as alunas dos cursos da área de saúde, humanas e exatas e também se havia um aumento no conhecimento sobre o assunto durante o curso de graduação, comparando as alunas do primeiro e do último período de cada área.

Considerando apenas as respostas corretas para as questões $1,2,3,4$ e 5 comparamos, na Tabela 1, as diferenças entre as respostas das acadêmicas das áreas de saúde, humanas e exatas, e também por períodos.

O estudo identificou 178 relatos de possíveis eventos adversos aos contraceptivos orais apresentados pelas acadêmicas usuárias destes medicamentos, sendo que $26,4 \%$ de inchaço, 24,7\% de enxaqueca, 23,6\% de náuseas, $12,4 \%$ de aumento de peso, 5,6\% de perda do libido, 3,9\% de secreção mamária e $3,4 \%$ de manchas escuras no rosto.

Entre os contraceptivos orais mais utilizados destacam-se os que são compostos por acetato de ciproterona (2mg) eetinilestradiol $(0,035 \mathrm{mg})$, correspondendo a $29,0 \%$. Dentro deste grupo o medicamento mais relatado foi o Diane ${ }^{\circledR} 35$ do laboratório Bayer S.A. com 59,5\%.

Notou-se que o uso de minipílulas ainda não era convencional entre as universitárias, representado somente $\quad 0,7 \%$. 
Tabela 1 - Conhecimento das acadêmicas sobre os contraceptivos orais. Alfenas - MG, 2010.

\begin{tabular}{|c|c|c|c|c|}
\hline \multirow{2}{*}{ PERGUNTAS } & ÁREA & Primeiro Período & Último Período & Média Geral \\
\hline & & Não (\%) & Não (\%) & $(\%)$ \\
\hline \multirow{3}{*}{$\begin{array}{l}\text { 1. Você acredita que o } \\
\text { anticoncepcional oral engorda? }\end{array}$} & Saúde & 69,2 & 71,7 & 70,5 \\
\hline & Humanas & 73,9 & 82,4 & 78,1 \\
\hline & Exatas & 75,0 & 73,3 & 74,2 \\
\hline \multirow{4}{*}{$\begin{array}{l}\text { 2. Em sua opinião o uso da pílula } \\
\text { pode causar alguma dificuldade para } \\
\text { engravidar? }\end{array}$} & & Não (\%) & Não (\%) & $(\%)$ \\
\hline & Saúde & 71,8 & 52,2 & 62,0 \\
\hline & Humanas & 65,2 & 88,2 & 76,7 \\
\hline & Exatas & 64,3 & 73,3 & 68,8 \\
\hline \multirow{4}{*}{$\begin{array}{l}\text { 3. Em sua opinião é necessário } \\
\text { proporcionar um descanso da pílula } \\
\text { ao seu organismo? }\end{array}$} & & Não (\%) & Não (\%) & $(\%)$ \\
\hline & Saúde & 56,4 & 54,3 & 55,4 \\
\hline & Humanas & 56,5 & 58,8 & 57,7 \\
\hline & Exatas & 46,4 & 33,3 & 39,9 \\
\hline \multirow{4}{*}{$\begin{array}{l}\text { 4. Quando você tem vômitos ou } \\
\text { diarreia, você acha que a efetividade } \\
\text { do anticoncepcional diminui? }\end{array}$} & & $\operatorname{Sim}(\%)$ & $\operatorname{Sim}(\%)$ & $(\%)$ \\
\hline & Saúde & 53,8 & 65,2 & 59,5 \\
\hline & Humanas & 17,4 & 52,9 & 35,2 \\
\hline & Exatas & 39,3 & 53,3 & 46,3 \\
\hline \multirow{4}{*}{$\begin{array}{l}\text { 5. Assinale quais classes de } \\
\text { medicamentos } \\
\text { analgésicos, antibióticos, } \\
\text { antifúngicos e laxantes) podem } \\
\text { interferirna ação de alguns } \\
\text { contraceptivos orais. }\end{array}$} & & $\begin{array}{c}\text { Todas as } \\
\text { alternativas }(\%)\end{array}$ & $\begin{array}{c}\text { Todas as } \\
\text { alternativas }(\%)\end{array}$ & $(\%)$ \\
\hline & Saúde & 18,6 & 19,8 & 19,2 \\
\hline & Humanas & 20,0 & 20,0 & 20,0 \\
\hline & Exatas & 19,8 & 20,0 & 19,9 \\
\hline \multirow{4}{*}{$\begin{array}{c}\text { Média de respostas CORRETAS } \\
(\%)\end{array}$} & & Primeiro Período & Último Período & Média Geral \\
\hline & Saúde & 54,0 & 52,6 & 53,3 \\
\hline & Humanas & 46,6 & 60,5 & 53,5 \\
\hline & Exatas & 49,0 & 50,6 & 49,8 \\
\hline
\end{tabular}

Fonte: os autores

\section{DISCUSSÃO}

A prevalência de uso dos métodos anticoncepcionais nos dias atuais é cada vez mais alta. Estudo feito com adolescentes mostrou que $75 \%$ das entrevistas utilizavam algum método anticoncepcional (DUARTE et al., 2011). Em outro estudo foi constatado que $36,7 \%$ utilizavam o método anticoncepcional oral, e $26,7 \%$ utilizavam o método anticoncepcional oral associado ao preservativo masculino (PENAFORTE, et al., 2010). Fato que se confirma neste trabalho.
Ainda hoje, as usuárias dos $\mathrm{CO}$ utilizam em maior quantidade justamente para evitarem uma gravidez indesejada, mas também observado que o uso desses medicamentos foram utilizados para regulação do ciclo menstrual e para o tratamento de acnes. Fato que também foi evidenciado por outro trabalho que cita um $\mathrm{CO}$, que além de evitar a gravidez e regular o ciclo hormonal também diminui a intensidade do fluxo menstrual e trata acne moderada e 
distúrbios de humor relacionados ao ciclo menstrual (NUCCI, 2012).

Quanto aos cuidados sobre a possível efetividade da pílula observou-se que as acadêmicas possuem cuidados quanto ao modo de usar esses medicamentos sendo que a grande maioria administravam no mesmo horário, no entanto apenas um pouco mais da metade das acadêmicas não relataram esquecimento de tomar nenhum comprimido da cartela. Além do esquecimento ou da pontualidade na administração dos $\mathrm{CO}$ sabese que o estado de saúde da usuária, como por exemplo, vômito e diarreia influenciam na efetividade do medicamento (BRASIL, 2013a).

Em um estudo com estudantes ingressantes de uma universidade pública de São Paulo de áreas da saúde, exatas e humanas observou-se que $70,4 \%$ acreditavam que as mulheres não precisavam utilizar um método de barreira até o próximo ciclo menstrual caso tenham tido algum quadro de diarreia ou vômitos por mais de 24 horas (ALVES, et al., 2008). No presente estudo observaram-se dados parecidos, principalmente com as alunas dos primeiros períodos. Fato preocupante, visto que vômitos e diarreias são sintomas comuns de diversas enfermidades que podem acometer a todo instante essas jovens e gerar um possível gravidez indesejada.

Já quando questionadas sobre se o CO causa dificuldades para engravidar, grande parte das alunas da saúde acertaram a resposta dizendo que não, no entanto em um trabalho verificou-se dados semelhantes, onde $70,3 \%$ das estudantes de enfermagem e medicina entrevistadas disseram que a mulher que usa CO por muito tempo ficava protegida de uma gravidez quando parava de tomá-lo (SEABRA et al., 2012).

Ao serem questionadas sobre o efeito colateral de ganhar peso, os cursos possuíam um conhecimento semelhantes sobre a questão. Sabe-se que essa afirmação não passa de um mito, mas que, no entanto ainda é aderido pela sociedade muitas vezes pela pouca informação sobre tal assunto, como relata (TORRES, MOREIRA, 2005 citado por PATIAS, et al., 2012). Embora neste estudo tiveram relatos de evento adverso ao $\mathrm{CO}$ com relação ao aumento de peso.

No que diz respeito à segurança do uso dos $\mathrm{CO}$, percebeu que as alunas possuíam uma boa qualidade de vida, já que sabe-se que não é recomendado o uso de $\mathrm{CO}$ para pessoas com diabetes mellitus, insuficiência renal, presença ou história de processos trombóticos/tromboembólicos entre outros (BRASIL, 2013a). Além disso, o uso do cigarro pode levar a problemas cardíacos graves quando associados ao uso de $\mathrm{CO}$ (LEMOS et al., 2011). Também pode citar os acidentes vasculares celebrais que estão diretamente relacionados a hipertensão arterial e possui como fator de risco significativo o uso de anticoncepcional oral a base de estrogênios (POLLOCK, WILMORE, 1993 citado por ROSA et al., 2006). 
Todas as áreas apresentaram baixa confiança no CO. No entanto, o uso deste deve ser feito de forma correta e quando isso é seguido a taxa de gravidez chega a mínimos 0,5 a 1,0 por 100 mulheres/ano de risco (CHROUSOS et al., 2003 citado por SANTOS et al., 2006).

Quanto às possíveis reações adversas oriundas do uso do $\mathrm{CO}$, sintomas relatados em bulas de medicamentos, que traz náuseas e enxaquecas como reações adversas frequentes (BRASIl, 2013a).

O CO mais utilizado pelas estudantes foi Diane® 35, que em janeiro de 2013 foi citado em um informe da agência de

\section{CONCLUSÃO}

Conclui-se não houve uma diferença considerável entre as diferentes áreas e nem entre os períodos. Apesar das universitárias apresentarem melhores condições de vivenciar a sexualidade de forma mais segura e sem riscos a sua saúde, o segmento mais escolarizado também se depara com inconsistências no uso de métodos contraceptivos.

Por isso a graduação deve ter ações voltadas não apenas para finalidades teóricas, mas também através de atividades educativas extracurriculares voltadas para a saúde da

\section{REFERÊNCIAS}

ALVES, A. S.; LOPES, M. H. B. M. Conhecimento, atitude e prática do uso de pílula e preservativo entre adolescentes universitários. medicamentos francesa (ANSM - Agence Nationale de Sécurité du Médicament et des Produits de Santé), alertando sobre os casos citados a partir da base de farmacovigilância, em que quatro mortes foram devido a trombose venosa relacionada com Diane ${ }^{\circledR} 35$. No Brasil, a Agência Nacional de Vigilância Sanitária responsável pela fiscalização dos medicamentos informou que na bula do medicamento em questão já possuía informações sobre a contraindicação do uso por mulheres com históricos trombóticos, infarto do miocárdio, acidente vascular cerebral entre outros (BRASIL, 2013b).

mulher independente da área. Mas em especial para área de saúde, uma vez que estes necessitam de informações sobre o assunto já que os profissionais de saúde devem estar preparados para lidar com mitos e conceitos errados sobre a utilização dos métodos anticoncepcionais.

\section{AGRADECIMENTO}

Ao PROBIC/UNIFAL-MG pelo apoio a pesquisa. Aos membros do Centro de Farmacovigilância da UNIFAL-MG (CEFAL) pela colaboração na pesquisa.

Revista Brasileira de Enfermagem, v. 61, n. 1, p. 11-17, 2008. Disponível em: http://www.scielo.br/pdf/reben/v61n1/02.pdf. Acesso em: 27 mai. 2013. 
BRASIL. Agência Nacional de Vigilância Sanitária - ANVISA. Bulário Eletrônico.

YAZ® Bayer S.A.2013a. Disponível em:http://www.anvisa.gov.br/fila_bula/frmVis ualizarBula.asp?pNuTransacao $=2903862013 \&$ pIdAnexo=1571724. Acesso em: 27 mai. 2013.

BRASIL. Agência Nacional de Vigilância Sanitária - ANVISA. Informe SNVS/Anvisa/Nuvig/GFARM n ${ }^{\circ}$ 01, de 29 de janeiro de 2013b. Disponível em:http://portal.anvisa.gov.br/wps/wcm/conn ect/95bd54804e5c8bdc930adfd762e8a5ec/Inf orme_Diane35_29012013.pdf?MOD=AJPER ES. Acesso em: 27 mai. 2013.

DUARTE, H. H.; BASTOS, G. A. N.; CORLETA, H. E. Utilização de métodos contraceptivos por adolescentes do sexo feminino da Comunidade Restinga e Extremo Sul. Rev Paul Pediatr, v. 29, n. 4, p. 572576, 2011. Disponível em: http://www.scielo.br/pdf/rpp/v29n4/16.pdf. Acesso em: 27 mai. 2013.

FRANCO, B. M.; ÉBOLI, E.; FREIRE, F. Brasileira inicia a vida sexual mais cedo, tem mais acesso a contraceptivos e menos filhos.

Pesquisa Nacional de Demografia e Saúde (PNDS), 2008. Disponível em: http://oglobo.globo.com/pais/mat/2008/07/03/p nds_brasileira_inicia_vida_sexual_mais_cedo_ tem_mais_acesso_contraceptivos_menos_filho s-547081065.asp. Acesso em: 20 abril 2013.

LEMOS, E. R. L.; MOURA, E. R. F.; GUEDES, T. G.; NOGUEIRA, P. S. F.; SOUZA, C. B. J. Uso de métodos anticoncepcionais por mulheres assistidas em área distrital e fatores determinantes. Revista de APS, v. 14, n. 4, p. 417-423, 2011. Disponível em: http://www.aps.ufjf.br/index.php/aps/article/v iewFile/1187/553. Acesso em: 27 mai. 2013.

Martins, L. B. M.; Costa-Paiva, L.; Osis, M. J. D.; Sousa, M. D.; Pinto Neto, A. M.; Tadini, V. Conhecimento sobre métodos anticoncepcionais por estudantes adolescentes. Rev Saúde Pública, v. 40, n. 1, p. 57-64, 2006.
NUCCI, M. Seria a pílula anticoncepcional uma droga de "estilo de vida"? Ensaio sobre o atual processo de medicalização da sexualidade. Sexualidad, Salud y Sociedad Revista Latinoamericana, n. 10, p. 124-139, 2012. Disponível em:

http://www.scielo.br/scielo.php?pid=S198464872012000400006\&script=sci_arttext.

Acesso em: 27 mai. 2013.

PATIAS, N. D.; JAGER, M. E.; FIORIN, P. C.; DIAS, A. C. G. GESTAÇÕES NA ADOLESCÊNCIA: MULTIPLICIDADE DE EXPERIÊNCIAS EM UMA UNIDADE BÁSICA DE SAÚDE DE SANTA MARIA/RS. Revista Internacional Interdisciplinar INTERthesis, v. 9, n. 1, p. 260-277,2012. Disponível em: http://www.periodicos.ufsc.br/index.php/inter thesis/article/view/1807-1384.2012v9n1p260. Acesso em: 27 mai. 2013.

PENAFORTE, M. C. L. F.; SILVA, L. R.; ESTEVES, A. P. V. S.; SILVA, R. F.; SANTOS, I. M. M.; SILVA, M. D. B.Conhecimento, uso e escolha dos métodos contraceptivos por um grupo de mulheres de uma Unidade Básica de Saúde em Teresópolis, RJ. Cogitare Enfermagem, v. 15, n. 1, p. 124130, 2010. Disponível em: http://ojs.c3sl.ufpr.br/ojs2/index.php/cogitare/arti cle/view/17183/11318. Acesso em: 27 mai. 2013.

ROSA, C. M.; COUTINHO, K. S.; DOMINGUES, M. F. MOURA, D. S. A prevalência de acidente vascular cerebral (AVC) no pós-operatório de revascularizaçao do miocárdio. Revista Brasileira em

Promoção da Saúde, v. 19, n. 1, p. 25-34, 2006. Disponível em: http://ojs.unifor.br/index.php/RBPS/article/vie w/957. Acesso em: 27 mai. 2003.

SANTOS, M. V.; LOYOLA, G. S. I.; MORAES, M. L. C.; LOPES, C. L. A eficácia dos contraceptivos orais associados ao uso de antibióticos. Rev. Ciênc. Méd., v. 15, n. 2, p. 143-149, 2006. Disponível em: http://bases.bireme.br/cgibin/wxislind.exe/iah/online/?IsisScript=iah/ia '1.xis\&src $=$ google $\&$ base $=$ LILACS\&lang $=$ p \& 
nextAction $=$ lnk\&exprSearch $=467817 \&$ index Search=ID. Acesso em: 27 mai. 2013.

SEABRA, L. O.; NERY, I. S.; MOREIRA, F. H. B.; ROCHA, J. S.; GONÇALVES, L. R. R. Conhecimento sobre métodos contraceptivos por universitários da área de saúde. João Pessoa. Anais do $17^{\circ}$ Encontro Nacional da Rede Feminista Norte e Nordeste de Estudos e Pesquisa sobre a Mulher e Relações de Gênero, 15 p., 2012. Disponível em:http://www.ufpb.br/evento/lti/ocs/index.p hp/17redor/17redor/paper/view/328. Acesso em: 27 mai. 2013.

TRUSSELL J.; VAUGHAN, B. Contraceptive failure, method-related discontinuation and resumption of use: results from the 1995 National Survey of Family Growth. Fam Plann Perspect, vol. 31, n. 2, p.64-72, 1999. 
Revista da Universidade Vale do Rio Verde, Três Corações, v. 10, n. 1, p. 58-66, jan./jul. 2013 